\title{
Stereo Viewing 3-Component, Planar PIV Utilizing Fuzzy Inference
}

Mark P. Wernet

Lewis Research Center

Cleveland, Ohio

Prepared for the 19th Advanced Measurement and Ground Testing Technology Conference sponsored by the American Institute of Aeronautics and Astronautics New Orleans, Louisiana, June 17-20, 1996

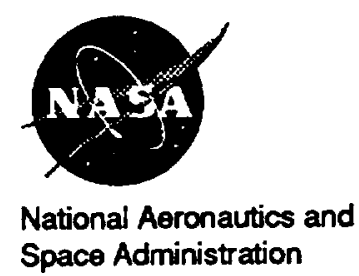




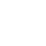

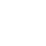




\title{
STEREO VIEWING 3-COMPONENT, PLANAR PIV UTILIZING FUZZY INFERENCE
}

\author{
Mark P. Wernet \\ National Aeronautics and Space Administration \\ Lewis Research Center \\ Cleveland, Ohio
}

\section{ABSTRACT}

An all electronic 3-D Digital Particle Image Velocimetry (DPIV) system has been developed for use in high velocity (supersonic) flows. Two high resolution $\mathrm{CCD}$ cameras mounted in a stereo viewing configuration are used to determine the out-of-plane velocity component from the difference of the in-plane velocity measurements. Double exposure image frames are acquired and Fuzzy inference techniques are used to maximize the validity of the velocity estimates obtained from the auto-correlation analysis. The $\mathrm{CCD}$ cameras are tilted relative to their respective lens axes to satisfy Scheimpflug's condition. Tilting the camera film plane ensures that the entire image plane is in focus. Perspective distortion still results, but can be corrected by proper calibration of the optical system. A calibration fixture is used to determine the experimental setup parameters and to assess the accuracy to which the $z$-plane displacements can be estimated. The details of the calibration fixture and procedure are discussed in the text. A pair of pulsed Nd:YAG lasers operating at $532 \mathrm{~nm}$ are used to illuminate the seeded flow from a convergent nozzle operated in an underexpanded condition. The light sheet was oriented perpendicular to the nozzle flow, yielding planar cross-sections of the 3-component velocity field at several axial stations. The key features of the supersonic jet are readily observed in the cross-plane vector plots.

\section{INTRODUCTION}

PIV is a widely used technique for measuring the inplane two-component velocity field across a planar region of a flow field illuminated by a pulsed laser light sheet. Particles entrained in the fluid scatter light which is collected normal to the plane of the light sheet. The collected light is imaged onto a photographic plate or a CCD camera. Multiple images of the particles are recorded via short time intervals between laser light sheet pulses. The exposures may be recorded either on a single photographic plate or on a CCD image frame. When CCD cameras are used to record the collected light, single exposures may also be recorded on separate image frames enabling more flexibility in the subsequent data processing.

Multiple image frame recording can be processed via either cross-correlation, particle tracking or a combination of both techniques depending on the seed particle density. For high seed density flows correlation techniques are typically employed where the average velocity across a small subregion is computed. ${ }^{1,2}$ This process is repeated over a regular grid across the image frame yielding the velocity vector map. In low seed density cases, particle tracking techniques are employed to determine the velocity of individual particles on the image frame. ${ }^{3,4}$ In some instances, both techniques can be used to improve on the spatial resolution of the resulting velocity vector map. ${ }^{5,6}$

In cases with multiple exposures recorded on a single image frame the data processing must be done using an auto-correlation technique. Again the average velocities across small sub-regions of the flow are determined over a regular grid. The auto-correlation operation places certain restrictions on the minimum displacement of the recorded particle images between exposures, therefore reducing the dynamic range. ${ }^{1}$

Although PIV has made 2-component planar measurements rather commonplace, many fluid engineering problems of interest are fully three dimensional and therefore do not possess a plane of symmetry for easy 2-component, planar measurements. Many approaches have been proposed and investigated for obtaining 3-component velocity information utilizing the PIV technique. These approaches can be broken into two separate classes: 3-component velocity measurements in a volume; and 3-component velocity measurements across a plane. Most of the volumetric approaches are limited to tracking a relatively low number of particles in the volume and suffer from particle overlap ambiguities. ${ }^{7.9}$ Holographic techniques have also been proposed and demonstrated for volume 3-component velocity estimates via particle tracking. ${ }^{10}$ Barnhart, Adrian, et. al. have demonstrated a holographic PIV system employing high seed particle density and correlation data reduction. ${ }^{.1}$ 
The 3-component planar measurement region techniques are simply an extension of the in-plane 2component PIV technique. In the planar approach, multiple recording cameras in a stereo viewing configuration or creative techniques for combining multiple views on a single camera are used. ${ }^{12-14}$ There are two primary configurations for stereo PIV: lens translation; and angular displacement, as shown in figure $1 \mathrm{a}$ and $\mathrm{b}$. Lens translation is simple and for small field angles results in no appreciable optical distortion, but the common image area from the two cameras may be restrictively small unless the field angles are enlarged. ${ }^{13,15,16}$ The use of large field angles in the displacement technique increases the sensitivity to the out-of-plane displacement, but will also produce perspective distortion in the image. ${ }^{17}$

The angular displacement technique is considered to be more accurate; however, this technique results in perspective distortion on the image plane and typically requires a large depth of field to avoid defocusing across the image plane. ${ }^{10,13}$ The image plane of best focus does not fall on the straight back camera film plane, resulting in blurring at the edges of the image, see figure $1 b$. The use of high $f /$ number collection optics increases the depth of field and minimizes the blurring. The errors of perspective distortion can be ignored by keeping the coupling angle between the cameras small. However, sensitivity to the out-of-plane component is then reduced. ${ }^{18,19}$ A back reflection technique has also been proposed to completely remove the perspective distortion. ${ }^{10}$

The large depth of field requirement of the angular displacement technique is easily eliminated by tilting the film plane such that the Scheimpflug condition is satisfied. ${ }^{10,17.20}$ The Scheimpflug condition requires that the image plane, object plane and the median plane through the lens all intersect at a common point, see figure 2. The tilted object plane is imaged onto a tilted image plane where all points are in focus and the depth of field requirements of the optical system are reduced. The object to image distances vary horizontally across the image, resulting in perspective distortion. Both the vertical and horizontal magnification vary as a function of horizontal position on the image plane. The resulting two camera views of a rectangular object are shown in figure 2. These distortions can be accounted for in the data processing. The advantages of the angular displacement technique are the large field of view and the improved accuracy in estimating the out-of-plane displacement. An indirect advantage of the stereo viewing configuration is the collection of the particulate scattered light from an angle other than $90^{\circ}$ from the scattering plane. The scattering efficiency reaches a minimum at $90^{\circ}$ collection for the case of $p$-polarized light and small $(\sim 1 \mu \mathrm{m})$ particles, therefore, the oblique collection angles collect more light than would be detected from a standard PIV setup.

In this work we will describe and demonstrate an angular displacement, stereo viewing, tilt-back camera optical system satisfying the Scheimpflug condition. The equations relating object to image space are derived and used to perform an error analysis on the performance of the technique. The calibration equipment and procedure will be described. A set of validation tests are performed to verify the accuracy of the displacement estimates. Auto-correlation PIV data reduction is used to process the acquired images and Fuzzy inference techniques are used to improve the quality of the processed velocity vector maps. ${ }^{21}$ Erroneous velocity vectors are corrected by using the Fuzzy inference engine to examine the secondary and lower amplitude peaks in the correlation plane and replace the erroneous vector with the correct velocity vector based on information from the surrounding velocity vectors. In this manner the correlation plane peak detection is treated as a pseudo particle tracking process where all of the peaks in the correlation plane are treated as candidate displacement peaks. Flow continuity rules govern the Fuzzy inference engine to correctly identify the true correlation displacement peak. An automatic thresholding operation is also demonstrated on the processed data to maximize the data quality. Finally, the system will be used to map a supersonic flow from an underexpanded jet using crossplane slices through the jet flow. The system is shown to perform well even in the presence of the large velocity gradient caused by the Mach disk in the underexpanded flow.

\section{OPTICAL SYSTEM}

An all electronic 3-component PIV system was required to satisfy the needs of expedient processing and convenient storage of the velocity data. Although high resolution CCDs $(1320 \times 1040)$ were to be used, the resultant field of view is still quite small when compared to that available from photographic plates. Therefore, the angular displacement method was selected for the optical configuration so as to maximize the field of view. The two drawbacks of the angular displacement technique are that a large depth of field is required to keep the object plane in focus and the image magnification varies with horizontal position on the image plane. The resulting image of a rectangular 
object is an isosceles trapezoid, see figure 2. By invoking the Scheimpflug condition, a technique commonly used in photography for image rectification, the large depth of field requirement is eliminated. The tilted object plane is exactly imaged onto the tilted image plane. Perspective distortion still remains in the image.

The amount of film plane tilt required varies depending on the object $\left(d_{o}\right)$ and image $\left(d_{i}\right)$ distances and the angular displacement half angle $\beta$. The total coupling angle between the cameras is $2 \beta$. The film plane tilt angle $\alpha$ is given by:

$$
\alpha=\tan ^{-1}\left[\frac{d_{i} \tan \beta}{d_{0}}\right]
$$

The transformation from object to image space can be readily developed by invoking the colinearity condition which requires that a ray connecting an object $\left(x_{o}, y_{o}\right)$ and image point $\left(x_{i}, y_{i}\right)$ passes through the center of the lens as shown in figure 2 . The object space coordinate system is centered at the intersection point of the two optical systems at the $z_{o}=0$ plane. The $y$-direction is out of the plane of the paper. Each camera film plane has a coordinate system centered about the middle of the film plane at $\left(x_{f p_{s}}, y_{f p_{t}}\right)$. Again on the film plane the $y$ coordinate is out of the plane of the page and $x$ lies in the plane. The resulting transformations for each camera view are:

$$
\begin{aligned}
& x_{i_{j}}=\frac{d_{i_{i}}\left(x_{o} \cos \beta_{j} \pm z_{o} \sin \beta_{j}\right)}{\left\{d_{o_{j}}-\left(z_{0} \cos \beta_{j} \mp x_{0} \sin \beta_{j}\right) \pm \tan \alpha_{j}\left[x_{0} \cos \beta_{j} \pm z_{o} \sin \beta_{j}\right]\right\}}+x_{j p_{1}} \\
& y_{i_{1}}=\frac{d_{i_{j}} y_{0}}{\left\{d_{o,}-\left(z_{0} \cos \beta_{j} \mp x_{0} \sin \beta_{j}\right) \pm \tan \alpha_{j}\left[x_{0} \cos \beta_{j} \pm z_{0} \sin \beta_{j}\right]\right\}}+y_{j p_{j}}
\end{aligned}
$$

where $j=1,2$ corresponds to the two camera views, and the top symbol in the \pm corresponds to view \#1 and the bottom symbol to view \#2. A more useful form of the equations would be to express the object plane displacements in terms of the two measured image plane displacements (one set from each camera). The next few steps are designed to achieve the desired result. If we assume the particle moves to the new object plane location $x_{0}+d x, y_{0}+d y, z_{0}+d z$ then on the image plane we can write for camera \#1:

$$
x_{i_{1}}=\frac{d_{i_{1}}\left[x_{0} \cos \beta_{1}+z_{0} \sin \beta_{1}\right]}{d_{o_{1}}-z_{0} A_{1}+x_{0} C_{1}}+x_{f p_{1}}
$$

$$
x_{i_{1}}+d x_{i_{1}}=\frac{d_{i_{1}}\left[\left(x_{0}+d x\right) \cos \beta_{1}+\left(z_{o}+d z\right) \sin \beta_{1}\right]}{d_{o_{1}}-\left(z_{o}+d z\right) A_{1}+\left(x_{o}+d x\right) C_{1}}+x_{f p_{1}}
$$

where $\quad C_{1}=\sin \beta_{1}+\tan \alpha_{1} \cos \beta_{1}$, $A_{1}=\cos \beta_{1}-\tan \alpha_{1} \sin \beta_{1}$ and $d x_{i j}$ is the perceived displacement on the camera sensor surface.

Now by subtracting equation 4 from equation 5 and assuming terms of order $d z^{2}, d x^{2}, d z d x \ll d_{o_{1}}$ we find:

$$
\begin{array}{r}
d x_{i_{1}}=M_{1}\left\{d x \cos \beta_{1}\left[1-\left(x_{0}+z_{0} \tan \beta_{1}\right) \frac{M_{1} C_{1}}{d_{i_{1}}}\right]+\right. \\
\left.d z \sin \beta_{1}\left[1+\left(z_{0}+\frac{x_{0}}{\tan \beta_{1}}\right) \frac{M_{1} A_{1}}{d_{i_{1}}}\right]\right\} \\
d y_{i_{1}}=M_{1}\left\{d y+d z \cdot y_{0} \frac{M_{1} A_{1}}{d_{i_{1}}}-d x \cdot y_{0} \frac{M_{1} C_{1}}{d_{i_{1}}}\right\}
\end{array}
$$

and similarly for camera 2 :

$$
\begin{array}{r}
d x_{i_{2}}=M_{2}\left\{d x \cos \beta_{2}\left[1+\left(x_{0}-z_{0} \tan \beta_{2}\right) \frac{M_{2} C_{2}}{d_{i_{2}}}\right]-\right. \\
\left.d z \sin \beta_{2}\left[1+\left(z_{0}-\frac{x_{0}}{\tan \beta_{2}}\right) \frac{M_{2} A_{2}}{d_{i_{2}}}\right]\right\} \\
d y_{i_{2}}=M_{2}\left\{d y+d z \cdot y_{0} \frac{M_{2} A_{2}}{d_{i_{2}}}+d x \cdot y_{0} \frac{M_{2} C_{2}}{d_{i_{2}}}\right\}
\end{array}
$$

where $\quad C_{2}=\sin \beta_{2}+\tan \alpha_{2} \cos \beta_{2}$,

$A_{2}=\cos \beta_{2}-\tan \alpha_{2} \sin \beta_{2}$, and the optical system magnifications are:

and

$$
M_{1}=\frac{d_{i_{1}}}{d_{o_{1}}-z_{o} A_{1}+x_{o} C_{1}}
$$

$$
M_{2}=\frac{d_{i_{2}}}{d_{o_{2}}-z_{0} A_{2}-x_{0} C_{2}} .
$$


These equations describing the recorded particle displacements on the image planes can be inverted to get the explicit expressions describing the particle displacements in the vicinity of the object plane $\Delta x, \Delta y$, $\Delta z$ :

$\Delta z=$

$$
\frac{\frac{d x_{1}}{M_{1}}-\frac{d x_{2}}{M_{2}} \gamma}{\sin \beta_{1}\left[1+\left(z_{0}+\frac{x_{0}}{\tan \beta_{1}}\right) \frac{M_{1} A_{1}}{d_{i}}\right]+\sin \beta_{2}\left[1+\left(z_{0}-\frac{x_{0}}{\tan \beta_{2}}\right) \frac{M_{2} A_{2}}{d_{i_{2}}}\right] \gamma}
$$$$
\Delta x=\frac{\frac{d x_{2}}{M_{2}}+\Delta z \sin \beta_{2}\left[1+\left(z_{0}-\frac{x_{0}}{\tan \beta_{2}}\right) \frac{M_{2} A_{2}}{d_{i_{2}}}\right]}{\cos \beta_{2}\left[1+\left(x_{0}-z_{0} \tan \beta_{2}\right) \frac{M_{2} C_{2}}{d_{i_{2}}}\right]}
$$

$$
\Delta y=\frac{d y_{1}}{M_{1}}-\Delta z \cdot y_{0} \frac{M_{1} A_{1}}{d_{i_{1}}}+\Delta x \cdot y_{0} \frac{M_{1} C_{1}}{d_{i_{1}}}
$$

where:

$$
\gamma=\frac{\cos \beta_{1}\left[1-\left(x_{o}+z_{o} \tan \beta_{1}\right) \frac{M_{1} C_{1}}{d_{i_{1}}}\right]}{\cos \beta_{2}\left[1+\left(x_{o}-z_{o} \tan \beta_{2}\right) \frac{M_{2} C_{2}}{d_{i_{1}}}\right]}
$$

These equations describe the actual particle displacements in the vicinity of the object plane in terms of the measured displacements on the tilted CCD sensor surfaces in cameras 1 and 2 and the location of the particle in the object space. Equations 2 and 3 above can also be inverted so that the two stereo camera image coordinates $\left(x_{i}, y_{i}\right) \quad(j=1,2)$ of a selected object plane point $\left(x_{o}, y_{o} z_{0}\right)$ can be calculated.

\section{ERROR ANALYSIS}

The equations above describing the physical particle displacements in terms of the two in-plane PIV displacements can be used to determine the errors in the measured velocity components. The computed displacements depend on many physical parameters in the optical setup which will be ignored in the analysis, since their values can be determined to reasonable accuracy and their effect should be minimal. Assuming that both optical systems are identical $\left(\beta_{l}=\beta_{2}, \alpha_{1}=\alpha_{2}\right.$, and $d_{i}$ and $d_{o}$ from both camera views are identical) the errors in $\Delta z, \Delta x$ and $\Delta y$ are given below:

$$
\begin{aligned}
& \sigma_{s 2}^{2}= \\
& \frac{\delta_{\text {至 }}^{2}}{\left[M_{1} \sin \beta\left\{\left[1+\left(z_{0}+\frac{x_{o}}{\tan \beta}\right) \frac{M_{1} A}{d_{i}}\right]+\left[1+\left(z_{0}-\frac{x_{0}}{\tan \beta}\right) \frac{M_{2} A}{d_{1}}\right] \gamma\right\}\right]^{2}}+ \\
& \frac{\delta_{\alpha, 1}^{2} \gamma^{2}}{\left[M_{2} \sin \beta\left\{\left[1+\left(z_{0}+\frac{x_{0}}{\tan \beta}\right) \frac{M_{1} A}{d_{1}}\right]+\left[1+\left(z_{0}-\frac{x_{0}}{\tan \beta}\right) \frac{M_{2} A}{d_{i}}\right] \gamma\right\}\right]^{2}}+ \\
& {\left[\delta_{x} \frac{\partial \Delta z}{\partial x}\right]^{2}+\left[\delta_{:} \frac{\partial \Delta z}{\partial z}\right]^{2}} \\
& \sigma_{\Delta x}^{2}=\frac{\delta_{d x_{i}}^{2}}{\left\{M_{1} \cos \beta\left[1-\left(x_{0}+z_{0} \tan \beta\right) \frac{M_{1} C}{d_{i}}\right]\right\}^{2}}+ \\
& {\left[\frac{-\tan \beta\left[1+\left(z_{0}+\frac{x_{0}}{\tan \beta}\right) \frac{M_{1} A}{d_{i}}\right] \sigma_{\Delta z}}{\left[1-\left(x_{0}+z_{0} \tan \beta\right) \frac{M_{1} C}{d_{i}}\right]}\right]^{2}+} \\
& {\left[\delta_{x} \frac{\partial \Delta x}{\partial x}\right]^{2}+\left[\delta_{z} \frac{\partial \Delta x}{\partial z}\right]^{2}} \\
& \sigma_{\Delta y}^{2}=\delta_{y}^{2}\left[\Delta x \frac{M_{1} C}{d_{i}}-\Delta z \frac{M_{1} A}{d_{i}}\right]^{2}+\left[\frac{\delta_{d y_{1}}}{M_{1}}\right]^{2}+ \\
& {\left[\sigma_{\Delta x} y_{o} \frac{M_{i} C}{d_{i}}\right]^{2}+\left[\sigma_{\Delta z} y_{o} \frac{M_{1} A}{d_{i}}\right]^{2}}
\end{aligned}
$$

where $\delta_{d x l}, \delta_{d x 2}, \delta_{d y l}$ are the errors in determining the correlation peak locations, and $\delta_{x}, \delta_{y}, \delta_{z}$ are the errors in the location of the correlation subregion on the object plane. Some of the partial derivatives have not been explicitly shown due to their unruly size. The errors for $\Delta x, \Delta y$ and $\Delta z$ are plotted in figure 3 over a range of coupling half angles $\beta$ for the case $y_{o}=0$ and $z_{0}=0$. The independent variable is $x_{o}$. The errors in determining the correlation peak location were assumed to be $\delta_{d x j}=\delta_{d y j}=0.05 \varepsilon_{p} / M_{j}$, where $\varepsilon_{p}$ is the camera pixel size. 
Recall that the magnification is not constant in the image, hence the error will in general be a function of $x_{o}$ and $z_{0}$. Two estimates of $\Delta x$ and $\Delta y$ are available, one from each camera view. Only one of the two expressions for the error in $\Delta x$ and $\Delta y$ have been included above. In the computations of $\Delta x$ and $\Delta y$ the average of the two estimates from each camera view will be used.

Figure $3 \mathrm{a}$ shows that the error in $\Delta x$ changes very little with horizontal position, growing larger at the boundaries of the image and reaching a minimum at the center of the image. As the error coupling half-angle $\beta$ is increased, the error in $\Delta x$ increases. Figure $3 b$ shows the error in $\Delta y$, which is relatively insensitive to horizontal position on the object plane and the coupling half angle. Increasing the coupling half angle causes an increase in the error of $\Delta y$ only at the boundaries of the image. The errors remain relatively flat at the center of the image. Figure $3 \mathrm{c}$ shows the opposite trends from the previous two plots. Increasing the coupling half angle $\beta$ decreases the out-of-plane displacement error in $\sigma_{\Delta z}$. The error in $\Delta z$ is relatively insensitive to horizontal position on the image.

The major variables affecting the out-of-plane displacement $\Delta z$ in equation 10 above can be determined by making some further simplifying assumptions. In addition to the assumptions made above we can also assume that $\delta_{d x_{1}}=\delta_{d x_{2}}$ and $x_{o}=0$ then:

$$
\sigma_{\Delta z}=\frac{\delta_{d s}}{\sqrt{2} M \sin \beta}
$$

where $M=d_{i} / d_{o}$. Hence, we obtain the obvious result that the error in the out-of-plane displacement is directly related to the error in estimating the correlation peak location on the CCD sensor and inversely related to the product of the sine of the optical system coupling half angle and the optical system magnification. Simplifying equation 11 in a similar form and combining with equation 16 we find:

$$
\sigma_{\Delta x}=\frac{\sigma_{\Delta x}}{\tan \beta}
$$

This simplification allows us to define an approximate ratio of the error in $\Delta z$ to the error in $\Delta x$. For the case where $\beta=30^{\circ}$, the error in $\Delta z$ is 1.7 times as large as the in-plane error $\sigma_{\Delta x}$. At the limit of $\beta=45^{\circ}$, the error in the out-of-plane displacement equals the in-plane displacement error.
In the preceding analysis we assumed that there was no limitation on the minimum displacement that could be detected by each of the stereo viewing cameras. In reality only the cross-correlation technique possesses this property. Auto-correlation requires that the particles displace by at least their diameters so that the displacement peak can be distinguished from the zeroorder peak. If the flow velocity vector happens to point directly along the optical axis of one of the cameras, the auto-correlation technique will yield a "dead-band" region where particle displacements can not be detected. The "dead-band" feature of the autocorrelation technique has been ignored in the error analysis. The experiments below were designed so that the auto-correlation analysis would detect measurable displacements.

\section{CALIBRATION SYSTEM/OPTICAL HARDWARE}

The optical system is shown in figure 4. The cameras are mounted on carts with sliding dogs that fit into concentric radius grooves in the mounting table. The center location of the radial grooves is located 8 inches from the edge of the table. This configuration permits easy changes in the optical system coupling angle while maintaining a common focal point between the cameras. The lenses are mounted directly on the sliding carts and are directed normal to the radial grooves in the table. The cameras bodies are mounted independent of the lens on the sliding carts and are on rotatable bases so that the proper tilt angle $\alpha$ can be introduced. A flexible baffle is placed between the lens and the camera to prevent stray light from reaching the CCD sensor.

A calibration target consisting of an optical flat with a reticle grid on the front surface is mounted in a jig that connects to the camera mounting table to hold the target at center of rotation of the optical system. The reticle grid contains 5 horizontal and 5 vertical lines on $5 \mathrm{~mm}$ centers. The reticle is mounted on a 2-axis micrometer stage on the calibration jig support. The stages allow the calibration target to be moved to multiple object $z_{o^{-}}$ planes.

The optical system is setup so that the middle vertical and horizontal lines on the reticle are at the center of the camera system field of view. The images from the two cameras are electronically subtracted to aid in fine tuning the alignment. The center vertical and horizontal lines are made to overlap as best as possible with the 
calibration target at the $z_{0}=0$ plane. Calibration data are then taken at multiple $z_{0}$-planes $(0, \pm 0.5, \pm 1 \mathrm{~mm})$.

The calibration images are processed and used to determine the overall optical system setup parameters. The images are first processed using edge detection filters to find the horizontal and vertical lines on the ruled grid. The slopes and intercepts of all the lines (horizontal and vertical) are then determined in a global fitting operation. Knowledge about the vertical lines being parallel to each other and also perpendicular to the center horizontal line are incorporated into the fitting procedure. The slope of the center horizontal line is used to determine and later correct for the amount of rotation in each camera image about the optical axis. The extrapolated horizontal lines intersect at a point in space which is used to define a keystone transformation back to a Cartesian object space using just the slopes of either the top or bottom horizontal lines. ${ }^{22}$ The simple geometric keystone transformation is used to determine the optical system magnification at the center of the object plane $x_{o}=0, y_{o}=0, z_{0}=0$.

The intersection points of all of the lines in the calibration target for each camera view at all of the $z_{0}-$ planes are then used along with the optical system magnification (for each view) to fit for the other optical system parameters: $\alpha, \beta, d_{i}, d_{o}$, and the center coordinates of the object space on the $\mathrm{CCD}$ sensor plane $x_{f p_{j}}, y_{f p_{j}}$. A function minimization procedure is used to minimize the distances between the reconstructed object plane coordinates of the intersecting lines on the calibration target at the multiple $z$-planes and the known 3-D coordinates of the intersecting lines on calibration target at the mechanically displaced $z$-plane locations. The fit parameters constitute the stereo view optical system calibration allowing for the transformation from image to object space, or vice versa.

\section{EOUIPMENT AND DATA PROCESSING}

A pair of Nd:YAG lasers with $200 \mathrm{~mJ} /$ pulse energy at $532 \mathrm{~nm}$ are used to provide light sheet illumination. Two Kodak Megaplus 1.4 cameras are connected to EPIX framegrabber boards for data acquisition into a single PC. The camera resolution is $1320 \times 1040$ and the pixels are $6.8 \mu \mathrm{m}$ square with a $100 \%$ fill factor. Double exposure images are acquired with the illumination/recording system. The acquired image frames are processed via auto-correlation and a Fuzzy logic correlation peak detection algorithm is employed.
The image frames acquired with the angular displacement optical viewing system contain perspective distortion where a rectangular object becomes an isosceles trapezoid. The perspective distortion has two main components: an anamorphic component; and a shearing component. Anamorphic distortion manifests itself in a foreshortening of the $x$ dimension by approximately the cosine of the object plane tilt angle. The shearing distortion is caused by the variation in the $y$-plane magnification with horizontal position. The shear is largest at the upper and lower boundaries of the image and smallest along the horizontal centerline. In order to obtain a regular grid of velocity measurements on the object plane, the location and spacing of the image plane correlation subregions must vary with position. A regular grid of points is defined on the object plane and the corresponding set of image plane points for each camera view is computed and used to determine the center location of the correlation subregions. The computed velocity vectors are distorted by an amount dependent on their position on the image frame. On average, the anamorphic distortion for the $30^{\circ}$ viewing angle is approximately $15 \%$, while the shear is approximately $3 \%$. For small subregion sizes, the local distortion remains relatively constant across the subregion. The distortion is acceptable if it is constant across the subregion since the distortion is removed when the displacement vectors are mapped back to the object plane in the 3-D reconstruction process. Also, the 3-D reconstruction is initiated by constructing a planar grid in the object space from which the correlation subregion center locations are computed for each camera view. The average displacements across the subregions are computed and used to determine the out-of-plane displacements. Hence, the reconstruction does not rely on accurately matching the object plane coordinates of two image plane points (i.e. particle images) to estimate the out-of-plane displacement. Rather the out- of-plane displacement is computed from the difference of the average in-plane displacement over an extended subregion in each camera view, which is only sensitive to the order of the size of the subregion.

During the course of the auto-correlation data reduction software development, the choice of the image threshold level applied prior to auto-correlation processing was found to significantly affect the quality of the resulting vector map. The threshold level affects the amplitude and width of the zero-correlation peak and hence affects the ability of the correlation peak detection software to discern the first order particle displacement peak from the zero order peak. The variation in particulate seed across the image resulted in 
widely varying background noise levels. A high threshold level may produce good results in a high noise area, but will yield no correlation results in low noise regions of the image. An automatic threshold algorithm was found to yield good results over both high and low noise regions of the image. The auto-threshold technique simply computes the ratio of the number of non-zero pixels to the total number of pixels in each subregion. The threshold level is continuously increased until a ratio of $5 \%$ is achieved. The autocorrelation operation is then performed on the thresholded subregion.

The velocity vector map obtained using auto-correlation processing typically results in a few erroneous vectors in the velocity vector map. Many factors influence the ability of the data reduction software to accurately detect the true secondary peak corresponding to the average particle displacement across the subregion. ${ }^{6}$ For each subregion processed, the five largest peaks (other than the zero-order peak) on the correlation plane are detected and stored. After all of the subregions have been processed the Fuzzy inference engine is applied. At each subregion a set of velocity vectors are detected, which are then examined to determine the most probable displacement vector for that subregion. The velocity vectors in the surrounding subregions are also used in the evaluation of the candidate list at a given grid point. In this manner the correlation plane peak detection is treated as a pseudo particle tracking process where all of the peaks in each subregion's correlation plane are treated as candidate displacement peaks. Flow continuity rules govern the Fuzzy inference engine to correctly identify the true correlation displacement peak.

\section{VALIDATION TESTS}

Tests were performed to validate the capability of the stereo view optical system to accurately reconstruct the recorded three-dimensional displacements. The goal of the tests were twofold: first to determine if the optical system calibration was accurate; and second to determine if there were any residual effects of the perspective distortion in the reconstructed object field. Rather than use a computer simulation which neglects the effects of optical noise, digitization error, and lens distortion, the tests were designed to use as much of the actual experimental hardware as was feasible. The camera system was configured with a coupling half angle $\beta=30^{\circ}$. A card with a simulated field of randomly sized and spaced small white specks on a black background was mounted on the face of the calibration target reticle. The recorded images contain particles that are up to $4 \times 4$ pixels in size and there are approximately 8 to 10 particles per $32 \times 32$ subregion. The simulated particle field could then be moved via the 2 -axis translation stage so that the $z_{0}$ and $x_{0}$ positions of the simulated particle field could be altered between image recordings. The single exposure images were then electronically added to produce a double exposure image for each camera view.

The simulated particle field was moved through a variety of $\Delta x$ and $\Delta z$ displacements. The simulated double exposure images were then processed using the auto-correlation data processing software. The two velocity vector image maps obtained from the stereo views were then processed via the $3-D$ reconstruction software to compute the displacement field. Figure 5 shows the displacement field results from a particulate field mechanical displacement of $(0.5,0.0,0.3 \mathrm{~mm})$. The correlation subregions size was $64 \times 64$ with $50 \%$ overlap. The vector field plotted shows the $\Delta x$ and $\Delta y$ components of the displacement in the magnitude and angle of the vectors. The out-of-plane displacement $\Delta z$ is encoded in the color of the displacement vectors. Some spurious vectors are observed in the displacement map which resulted from low particulate seeding in these areas. The averages of the measured displacements over the entire field are: $\Delta x: 0.493 \pm$ $0.034 \mathrm{~mm}, \Delta y: 0.004 \pm 0.011 \mathrm{~mm}, \Delta z: 0.299 \pm 0.059$ $\mathrm{mm}$. The absolute value of the $\Delta y$ displacements were taken before computing the mean values to ensure that any symmetric distortions in the image did not cancel out. The results show that the measured displacements accurately reproduce the true mechanical displacement of the simulated particulate field. No residual effects from the image plane distortion are observed in the final reconstructed object plane. The subregion size used for this validation test are the largest that would be used to reduce the image frames, therefore, perspective distortion effects should be negligible. Computing the ratio of out-of-plane to in-plane errors yields $\sigma_{\Delta z} / \sigma_{\Delta x}=$ 1.7 , which agrees with the ratio predicted from the error analysis for an optical system coupling half angle of $\beta=30^{\circ}$.

\section{NOZZLE DATA}

The stereo view PIV system was next used to measure the 3-component velocity field of a nozzle jet flow. The nozzle centerline was directed parallel to the bisector of the stereo cameras as shown in figure 6 . This optical configuration allowed the measurement of cross sections of the nozzle flow at various downstream 
locations. Again the camera half angle was set to $\beta$ $=30^{\circ}$. The Nd:YAG laser beam was formed into a $2 \times 40$ $\mathrm{mm}$ sheet, which was directed from above the nozzle to a beam trap below.

The nozzle consisted of a constant radius contraction with an exit diameter of $6.35 \mathrm{~mm}$. The flow was seeded in the plenum section using a commercial six-jet atomizer filled with Rosco's smoke juice. The resulting mean seed particle size was approximately $1 \mu \mathrm{m}$. No ambient seed was introduced. The nozzle was operated at a plenum pressure of 75 psi, yielding an underexpanded supersonic flow. ${ }^{6}$ The flow from this nozzle has previously been characterized and contains a Mach disk approximately 1.5 nozzle diameters downstream of the nozzle exit plane. The flow upstream of the nozzle is approximately $630 \mathrm{~m} / \mathrm{s}$ and downstream of the Mach disk drops to $150 \mathrm{~m} / \mathrm{s}$. The flow then repeatedly ramps back up and shocks back down. Inter-frame exposure times from 0.8 to 1.2 microseconds were used to record the velocity field. The camera lenses were operated at $f / 10$ and the object and image distances were 322 and $47 \mathrm{~mm}$. The imaged area was approximately $60 \times 40 \mathrm{~mm}$.

Cross-plane measurements were obtained at five axial stations: $1.1,1.6,1.9,2.6$, and 3.0 nozzle diameters downstream. The images were processed with an autocorrelation subregion size of $32 \times 32$ with $50 \%$ overlap. Six exposure sets at each measurement station were acquired and the resulting velocity vector maps averaged. Figure 7 shows the resulting 3-component, planar cross sections of the measured nozzle flow, where the vector magnitudes are color coded. The vector plot at $z / d=1.1$ shows the supersonic accelerating flow. The cross sections at $z / d=1.6$ and 1.9 show the decelerated flow and the presence of the Mach disk. The Mach disk is denoted by the low velocity core flow containing the light green/yellow velocity vectors which is surrounded by the higher velocity (blue coded) outer flow. At $z / d=2.6$ the flow has ramped back up to supersonic and become more turbulent. The final station at $\mathrm{z} / \mathrm{d}=3.0$ shows again a decelerated flow which is also more turbulent than the measurements obtained closer to the nozzle exit plane.

\section{CONCLUSIONS}

An all electronic, angular displacement, stereo viewing 3-component PIV system has been described and demonstrated. The image plane focus was optimized by employing the Scheimpflug condition via a tilted camera back. An error analysis showed the expected errors in each of the measured displacements as a function of horizontal position on the object plane. Residual perspective distortion from the oblique viewing direction was demonstrated to be negligible for small $(32 \times 32)$ correlation subregions. The out-of-plane displacement error was shown to be approximately 1.7 times larger than the in-plane error for an optical system coupling half angle of $30^{\circ}$. Fuzzy inference techniques were used to analyze the correlation plane results to optimize the validity of the velocity vector maps. The stereo view PIV system was demonstrated on an underexpanded supersonic nozzle flow, where the light sheet was perpendicular to the flow direction. The system was shown to be sensitive to the large velocity gradients in the nozzle flow.

\section{REFERENCES}

1) Adrian, R. J., "Multi-Point Optical Measurements of Simultaneous Vectors in Unsteady Flow - A Review", Int. J. of Heat and Fluid Flow, Vol 7, pp127-145, 1986.

2) Willert, C. E. and Gharib, M., "Three-Dimensional Particle Imaging With a Single Camera", Experiments in Fluids, 12, pp353-358, 1992.

3) Wernet, M. P. and Pline, A., "Particle Image Velocimetry for the Surface Tension Driven Convection Experiment Using a Particle Displacement Tracking Technique", Fourth International Conference on Laser Anemometry, Cleveland, OH, pp 315-325, August 5-9, 1991.

4) Wernet, M. P., "Particle Displacement Tracking Applied to Air Flows", Fourth Inter-national Conference on Laser Anemometry, Cleveland, $\mathrm{OH}, \mathrm{pp}$ 327-335, August 5-9, 1991.

5) Keane, R. D., Adrian, R. J. and Zhang, Y., "Super-Resolution Particle Imaging Velocimetry", Meas. Sci. Technol., 6, pp754-768, 1995.

6) Wernet, M. P., "Fuzzy Inference Enhanced Information Recovery from Digital PIV Using Cross-Correlation Combined with Particle Tracking", SPIE Conference on Optical Diagnostics in Fluid and Thermal Flow, Vol 2546, San Diego, CA, July 9-14, 1995.

7) Adamczyk, A. A., and Rimai, L., "Reconstruction of a 3-Dimensional Flow Field From Orthogonal 
Views of Seed Track Video Images", Experiments in Fluids, 6, pp380-386, 1988.

8) Kobayashi, T., Saga, T., Sekimoto, K., "Velocity Measurement of Three-Dimensional Flow Around Rotating Parallel Disks by Digital Image Processing", FED-Vol. 85, Flow Visualization, pp 2936, December 10-15, 1989.

9) Okamoto, K., Hassan, Y. A., Schmidl, W. D., "Simple Calibration Technique Using Image Cross-Correlation For Three Dimensional PIV", FED-Vol. 209, Flow Visualization and Image Processing of Multiphase Systems, pp99-106, 1995.

10) Hinsch, K. D., "Three-Dimensional Particle Velocimetry", Meas. Sci. Technol., 6, pp742-753, 1995.

11) Barnhart, D. H., Adrian, R. J., Papen, G. C., "Phase-Conjugate Holographic System for High Resolution Particle Image Velocimetry", Appl. Opt., Vol 33, No. 30, pp7159-7170, 1994.

12) Grant, I., Fu, S., Pan, X., Wang, X., "The Application of an In-Line, Stereoscopic, PIV System to 3-Component Velocity Measurements", Experiments in Fluids, 19, pp214-221, 1995.

13) Gauthier, V. and Riethmuller, M. L., "Particle Image Velocimetry", von Karman Institute for Fluid Dynamics Lecture Series 1988-06, N8917179, March 21-25, 1988.

14) Willert, C. E. and Gharib, M., "Digital Particle Image Velocimetry", Experiments in Fluids, 10, pp181-193, 1991.

15) Prasad, A. K. and Adrian, R. J., "Stereoscopic Particle Image Velocimetry Applied to Liquid Flows", Sixth International Symposium on Applications of Laser Techniques to Fluid Mechanics, Lisbon, Portugal, July 20-23, pp6.1.1-6.1.8, 1992.

16) Arroyo, M. P. and Greated, C. A., "Stereoscopic Particle Image Velocimetry", Meas. Sci. Technol., 2, pp1181-1186, 1991.

17) Kingslake, R., Optics in Photography, SPIE Optical Engineering Press, 1992.

18) Funes-Gallanzi, M. and Bryanston-Cross, P. J., "Solid State Visualization of a Highly Three Dimensional Flow Using Stereoscopic Particle
Image Velocimetry (3DPIV)", SPIE Conference on Optical Diagnostics in Fluid and Thermal Flow, Vol 2005, San Diego, CA, pp 360-369, July 14-16, 1993.

19) Westerweel, J. and Nieuwstadt, F. T. M., "Performance Tests on 3-Dimensional Velocity Measurements with a Two-Camera Digital Particle-Image Velocimeter", Fourth International Conference on Laser Anemometry, Cleveland, OH, pp349-355, August 5-9, 1991.

20) Stephany, J. F. and Haas, W. E., "Keystone Compensation for Image Storage Systems", Appl. Optics, Vol 15, No. 6, pp 1626-28, 1976.

21) Wernet, M. P., "Fuzzy Logic Particle-Tracking Velocimetry", SPIE Conference on Optical Diagnostics in Fluid and Thermal Flow, Vol 2005, San Diego, CA, pp701-708, July 14-16, 1993.

22) Buchele, D., "Analytical Correction of Keystone Distortion", ADF Memorandum Task Order 4521, NASA Contract NAS3-25767, 1990. 

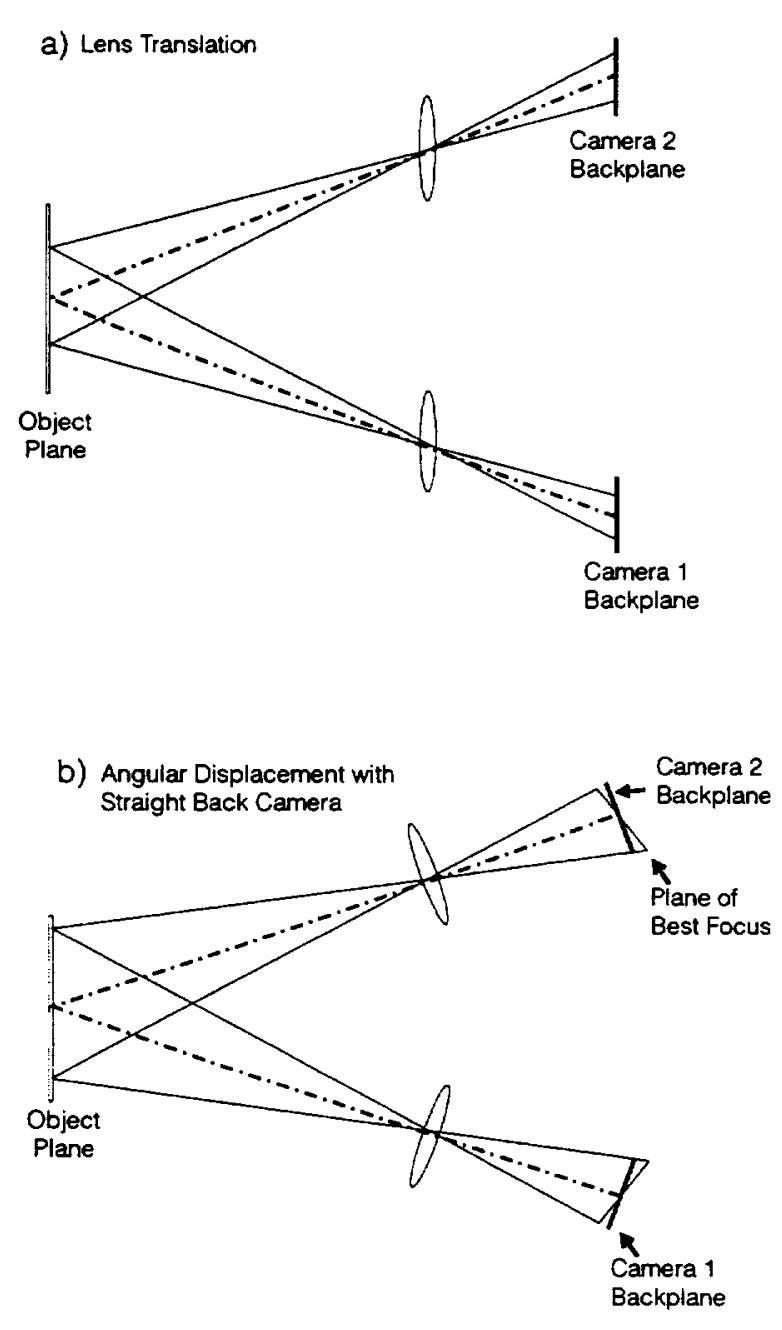

Figure 1: Schematic layouts of the two stereo view optical configurations: a) lens translation; b) angular displacement. The angular displacement cameras are shown with straight film backs. The plane of best focus does not lie on the film plane.

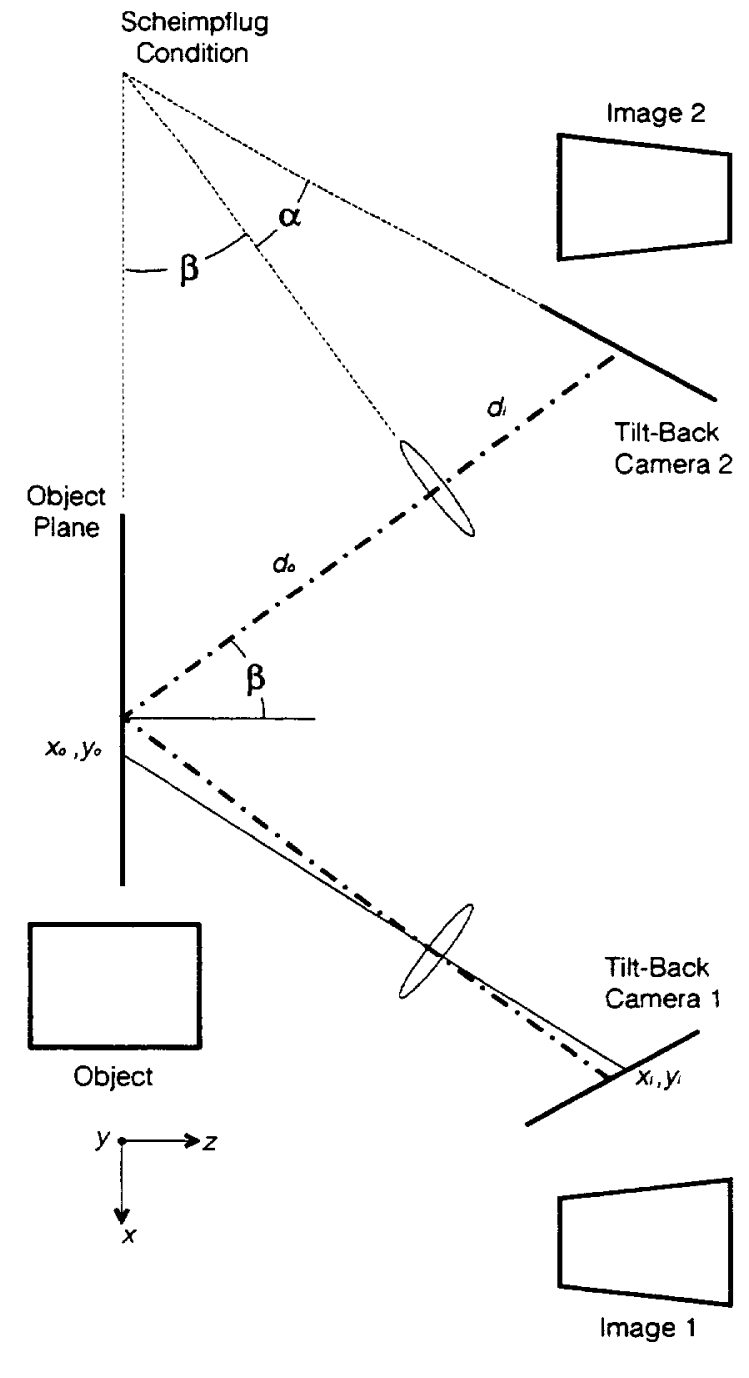

Figure 2: Optical configuration satisfying Scheimpflug's condition. The camera backs are tilted by an amount $\alpha$, and the angular displacement half angle is $\beta$. A rectangular object is shown to be imaged into an isosceles trapezoid. 


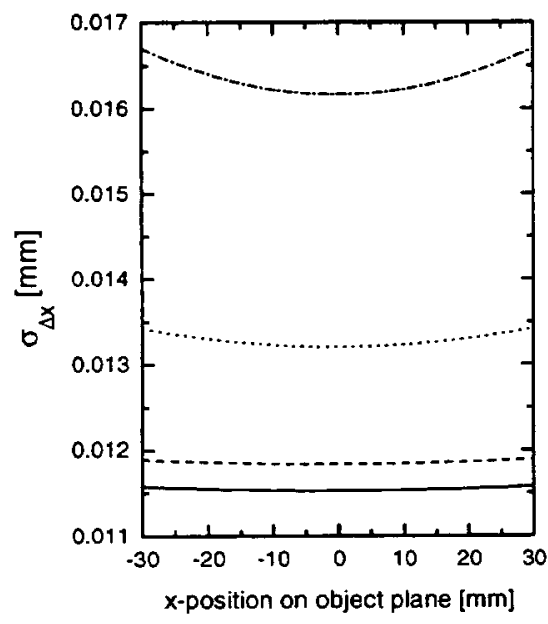

(a)

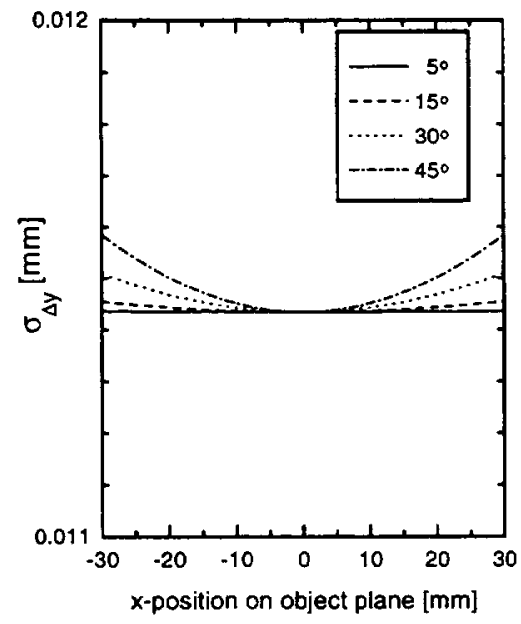

(b)

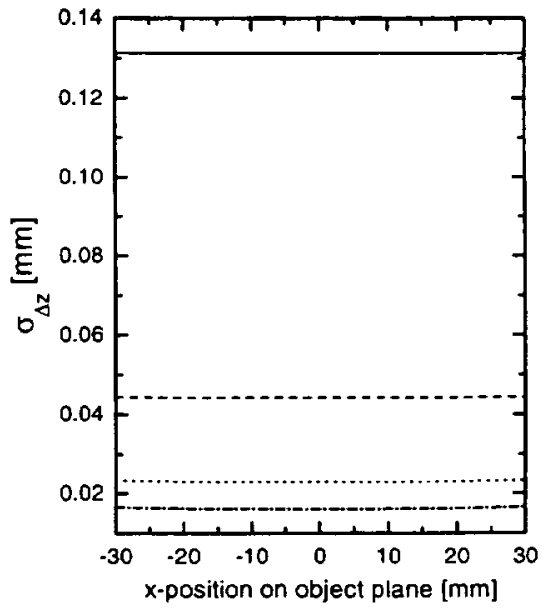

(c)

Figure 3: The error in $\Delta x, \Delta y$ and $\Delta z$ are plotted versus the horizontal position on the object plane. The errors are computed for a range of stereo camera coupling half angles $\left(\beta=5^{\circ}, 15^{\circ}, 30^{\circ}, 45^{\circ}\right)$. The errors are only weak functions of horizontal position. The errors in $\Delta x$ and $\Delta z$ are strong functions of the optical system coupling half angle. The error in determining the correlation peak location on the CCD sensor was assumed to be $\delta_{d x}=\delta_{d y}=0.05 \varepsilon_{p} / M$, where $\varepsilon_{p}$ is the camera pixel size.

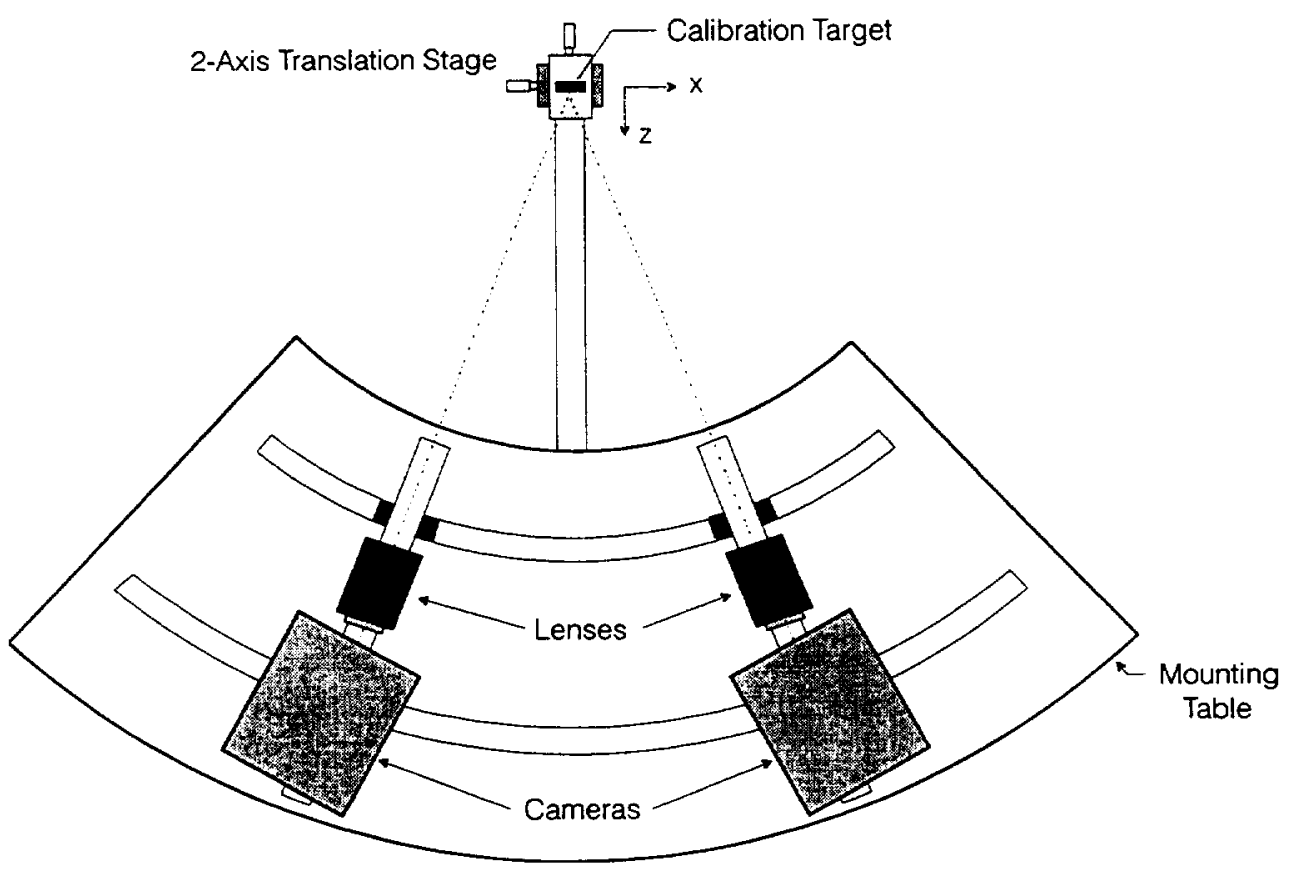

Figure 4: Orientation of optical hardware on the mounting table. The lens is mounted independent of the camera on sliding carts. The carts can be adjusted to change the coupling angle while maintaining a common focus point between the two cameras. The calibration target is shown on a two-axis translation stage. 


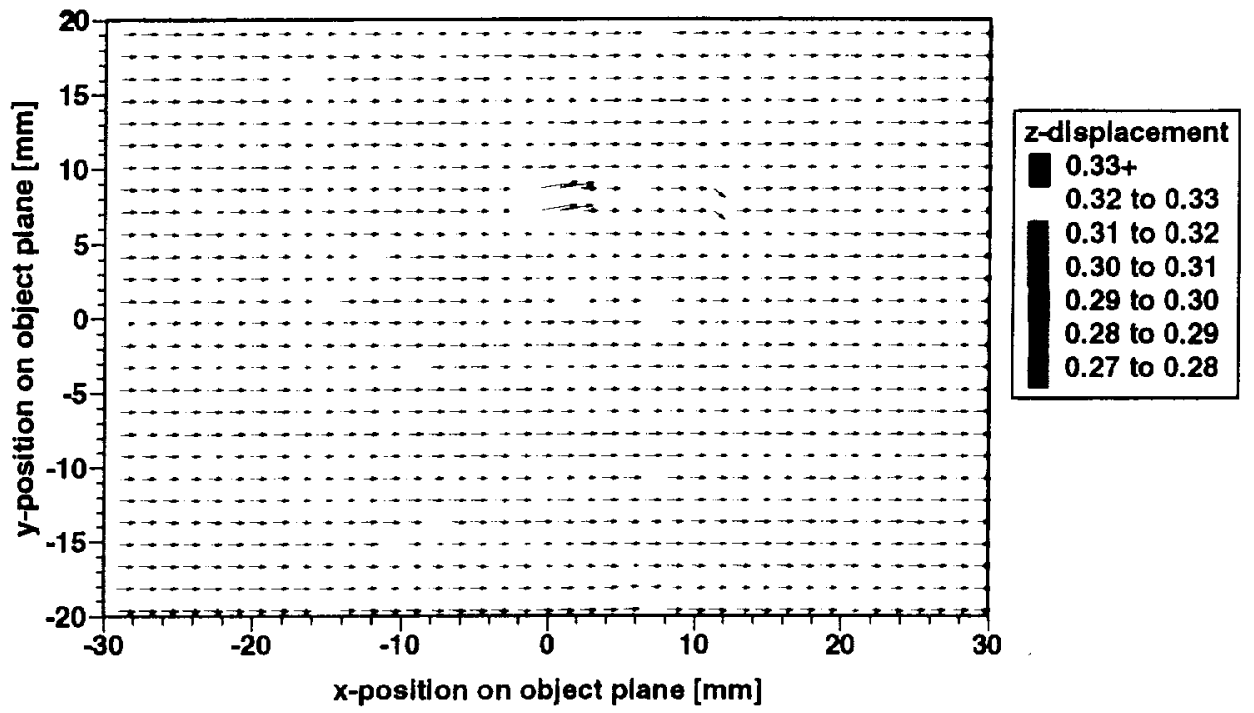
Mechanical Displacements:
Measurements:
$\Delta x: 0.5 \mathrm{~mm}$
$\Delta y: 0.0 \mathrm{~mm}$
$\Delta \mathrm{x}: 0.493 \pm 0.034 \mathrm{~mm}$
$\Delta z: 0.3 \mathrm{~mm}$
$\Delta y: 0.004 \pm 0.011 \mathrm{~mm}$
$\Delta z: 0.299 \pm 0.059 \mathrm{~mm}$

Figure 5: Validation test results for a mechanical translation of a simulated particle field. The in-plane displacements are depicted in the vector magnitude and angle. The out-of-plane displacements are encoded in the color of the vectors as shown by the accompanying key. The actual mechanical displacements and the average and standard deviation of the measured results across the plane are denoted in the figure. No residual effects from the perspective distortion are observed.

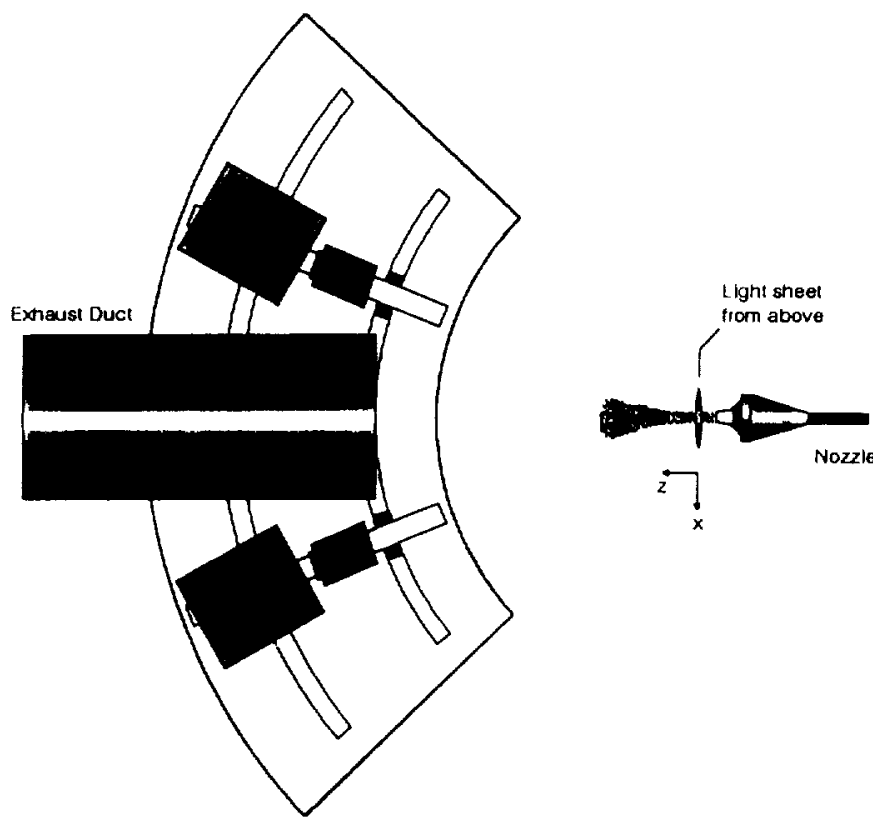

Figure 6: Experimental setup for the nozzle flow measurements. The light sheet is directed from above to a beam dump below the nozzle. An exhaust duct is mounted between the cameras to collect the seeded air stream. 


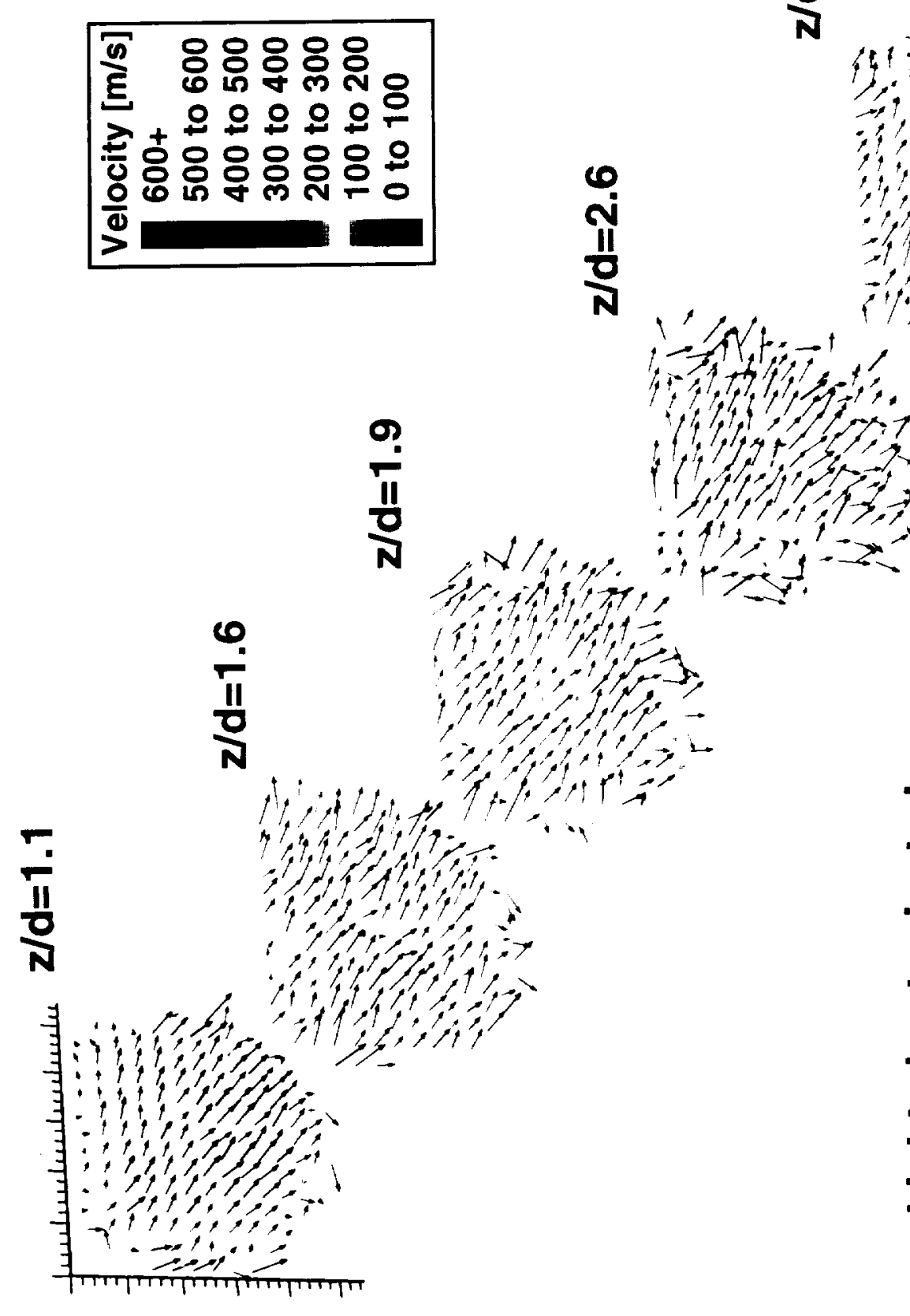

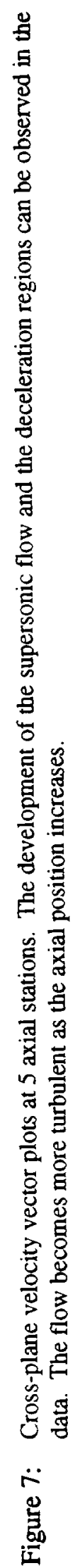




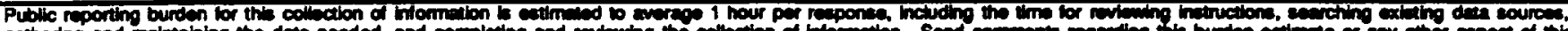
gesivering and in

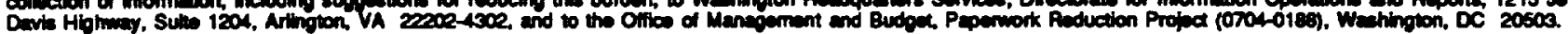

\begin{tabular}{|c|c|c|}
\hline 1. AGENCY USE ONLY (Leave blank) & $\begin{array}{l}\text { 2. PiEPOAT DATE } \\
\text { June } 1996\end{array}$ & $\begin{array}{l}\text { 3. FiEPOAT TYPE AND DATES COVERDD } \\
\text { Technical Memorandum }\end{array}$ \\
\hline
\end{tabular}

4. TIRE AND SUBTIIE

Stereo Viewing 3-Component, Planar PIV Utilizing Fuzzy Inference
5. FUVing NIMTERs

WU-505-62-50

6. Perforima ofchnization REPORT MUMBep

E-10278

10. SPONSOPINGANOWTOAING ACENCY REPORT MUMBER

NASA TM-107235

AIAA-96-2268

\section{SUPPLEMENTARY MOTES}

Prepared for the 19th Advanced Measurement and Ground Testing Technology Conference sponsored by the American Institute of Aeronautics and Astronautics, New Orleans, Louisiana, June 17-20, 1996. Responsible person, Mark P. Wernet, organization code 2520, (216) 433-3752.

122. DISTRABUTIONAVAlLABILTY STATEIENT 12b. DISTRIBUTION CODE

Unclassified -Unlimited

Subject Category 35

This publication is available from the NASA Center for AeroSpace Information, (301) 621-0390.

13. ABSTRACT (Maximum 200 words)

An all electronic 3-D Digital Particle Image Velocimetry (DPIV) system has been developed for use in high velocity (supersonic) flows. Two high resolution CCD cameras mounted in a stereo viewing configuration are used to determine the out-of-plane velocity component from the difference of the in-plane velocity measurements. Double exposure image frames are acquired and Fuzzy inference techniques are used to maximize the validity of the velocity estimates obtained from the auto-correlation analysis. The $\mathrm{CCD}$ cameras are tilted relative to their respective lens axes to satisfy Scheimpflug's condition. Tilting the camera film plane ensures that the entire image plane is in focus. Perspective distortion still results, but can be corrected by proper calibration of the optical system. A calibration fixture is used to determine the experimental setup parameters and to assess the accuracy to which the z-plane displacements can be estimated. The details of the calibration fixture and procedure are discussed in the text. A pair of pulsed Nd:YAG lasers operating at 532 $\mathrm{nm}$ are used to illuminate the seeded flow from a convergent nozzle operated in an underexpanded condition. The light sheet was oriented perpendicular to the nozzle flow, yielding planar cross-sections of the 3-component velocity field at several axial stations. The key features of the supersonic jet are readily observed in the cross-plane vector plots.

\begin{tabular}{|c|c|c|c|}
\hline \multicolumn{2}{|l|}{ 14. SUBJECT TERMS } & & $\begin{array}{c}\text { 15. MUMBER OF PAGES } \\
15\end{array}$ \\
\hline Particle tracking; Velocim & try & & $\begin{array}{r}\text { 16. PAICE COOE } \\
\text { A03 }\end{array}$ \\
\hline $\begin{array}{l}\text { 17. SECUATY CLASSIFICATION } \\
\text { OF REPORT } \\
\text { Unclassified }\end{array}$ & $\begin{array}{l}\text { 18. SECURTY CLASSIFICATION } \\
\text { OF THIS PAGE } \\
\text { Unclassified }\end{array}$ & $\begin{array}{l}\text { 19. SECURTY CLASSIFICATION } \\
\text { OF ABSTRACT } \\
\text { Unclassified }\end{array}$ & 20. LINTATION OF ABSTRACT \\
\hline
\end{tabular}




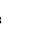




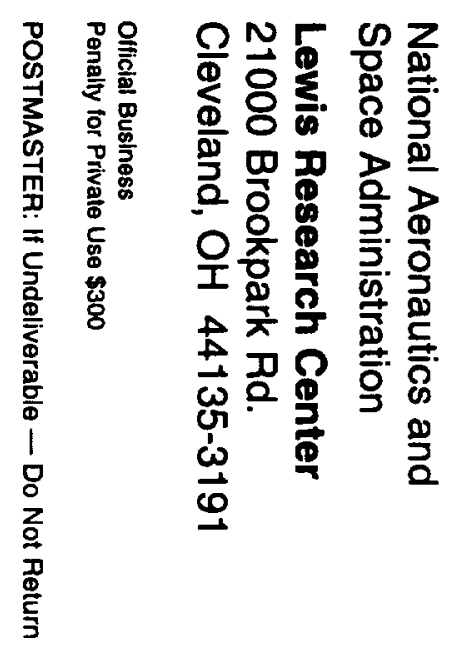

